the deformity and obtaining a useful $\operatorname{limb}$ for the patient, with bony anchylosis in a normal position; and to such a result $I$ would advise, in all future operations, that our expectations should be limited.

With regard to the subcutaneous operation, which, so far as I know, was first suggested and performed by myself, and which I have now brought before the British Medical Association, I would only observe that the subcutaneous division of bone-subcutaneous osteotomy, as it may be called - has provedd itself to be as simple and harmless an operation in its immediate effects as subcutaneous tenotomy, with which, in its essential characters, the operation may be compared.

Since this paper was read at the Association meeting, this operation has been successfully performed by Mr. T. R. Jessop of Leeds, who on the 17 th September, 1870 , writes as follows. "Three weeks ago last Thursday, I performed the operation upon a young woman whose right thigh was fixed by bony anchylosis at a right angle with the pelvis. By practising upon the dead subject, I had previously found that the best spot at which to make the puncture was about an inch behind the posterior margin of the great trochanter, near its upper border. The division we made without any difficulty. The operation and the subsequent dressing; were performed after Professor Lister's method. The wound healed at once without a single drop of pus. For a fortnight I kept the patient in bed, with the limb stretched by means of a weight slung over a pulley attached to the ankle. Since the expiration of a fortnight, I have had the woman up, walking about on crutches, and swinging the leg in all directions, with the view of procuring a false joint. The limb measures barely an inch shorter than the sound one; and the patient makes complaint only of a little pain in both hip and knee. Crepitus can both be felt and heard very distinctly. I will have the result duly reported." The operation has also been successfully performed by Mr. F. W. Jowers, of Brighton, and Mr. Furneaux Jordan, of Birmingham. No inflammation or suppuration followed the operation in either case. The details of these three succcessful cases will shortly be published; and the fact that this operation has been successfully performed in four cases, goes far to establish it as a surgical procedure.

\section{SUBCUTANEOLS SECTION OF THE NECK OF THE FEMUR.}

BY FURNEAUX JORDAN, F.R.C.S.,

Surgeon to the Queen's Hospital; Professor of Surgery in Queen's College. Birmingham; etc.

Emma H., aged ió, from Wales, had had hip-disease for six years. She had had several sinuses opening and closing during that time. On admission into hospital, there was a little onzing from one near the perinæum. The thigh was flexed at right angles to the trunk; and there was unmistakable osseous anchylosis of the hip-joint, as revealed by examination under chloroform. I divided the neck of the femur by the method and with the instruments devised by Mr. W. Adams. The sudden mobility of the joint when the section of the femur was completed was very striking. The adductor longus and long head of the rectus femoris required tenotomy. The limb was then put into a position which promises a very useful result. It is now three weeks since the operation, and the progress has been most favourable.

In this case, the naturally short femoral neck of early life, made shorter by caries and anchylosis, combined with a very fat gluteal region (since anchylosis, the patient has become very stout), required more than ordinary care in every step of the operation.

\section{ON SCARLET-FEVER, WITH SPECIAL REFERENCE TO PATHOLOGY AND TREATMENT.*}

By ROBERT RENFREW, M.D., Glasgow.

SCARLET FEVER is one of the zymotic diseases. These diseases are now generally believed to be produced by a specific organised substance entering the body, either by the stomach or lungs, or by both; when it has entered the system, it has the power of developing and multiplying itself, as seen in inoculation and vaccination. The process of multiplication deteriorates the blood, deranges the nervous system, quickens the circulation, causing rapid disintegration of the tissues, as evinced by the increased temperature, and altering the secretions from the secretory and excretory organs. Disintegration goes on with such

- Read in the Medicine Section before the Annual Meeting of the British Medical Association in Newcastle-upon-Tyne, August 1870 . rapidity that the normal physiological and chemical changes cannot take place, and disintegrated substances are floating in the blood, still further deteriorating the vital fluid.

The morbific matters of the zymotic diseases are not eliminated by the usual eliminating organs. Each poison is thrown off by a distinct and separate part of the body : small-pox, by the skin; cow-pox at the point of introduction; measles, by the mucous membranes of the eyes, nose, fauces, and lungs; hooping-cough, by the mucous membranes of the respiratory organs and stomach; enteric fever, by the lower third of the ileum ; cholera, by the stomach and bowels; scarlet fever, by the fauces, nose, and perhaps by the stomach. In typhus fever, the eliminating part is not so obvious, but, perhaps, it is done by the lungs. The parts specified above, however the poison may have been introduced into the blood, are always those affected. That the poison of enteric fever is thrown off by the intestines, and cholera by the intestines and stomach, there can be no doubt, for it has been proved that persons drinking water containing the discharges from these diseases have been sejzed with similar disorders.

It has been said that "it seems probable that in all varieties of scarlet fever a primary effect is suddenly and violently to stimulate the natural cell-growth of all the secreting organs." We have no evidence that this takes place anywhere else than in the parts pointed out; for in scarlet fever we have very seldom any disease of the lungs, liver, or bowels. Sometimes the kidneys show signs of congestion, but not more so than in pneumonia, typhoid, and typhus fever. In parts where we have always irritation or inflammation in zymotic diseases, I believe they are always caused by elimination or absorption of the poison.

The severity of the symptoms, whether general or local, in the zymotic class of diseases, depends upon the power of the system to develope the poison in the blood. For, if a number of persons be inoculated from the same person, there will be found a great variety in the number and character of the pustules. If several children be vaccinated with the same lymph, and with equal care as regards the number, depth, and extent of the scratches and quantity of matter applied, you will have a great diversity in the number of the vesicles and amount of lymph produced. In one child you may only have one vesicle, in another two or three, and so on, until you have a cluster of the size of a shilling.

At one time I thought that the small number of vesicles after vacoination depended on some imperfection in the introducing of the lymph, until I tried-when there was only one vesicle-vaccination on the other arm, and failed; showing that, however small the amount of lymph eliminated, the purpose had been accomplished, and that the power of multiplying the poison had been weak.

In every case of scarlet fever the throat is affected. The rash may be absent, but the throat-affection is never. In the mild form the fauces are red, somewhat swollen, and the secretion increased in quantity and altered in character. In scarlatina anginosa and maligna these appearances are all greatly aggravated, and they have extended themselves into the nose, and sometimes even into the Eustachian tube, causing great pain in the ear, and often suppuration. In these two forms the tonsils are found to be greatly enlarged, but in scarlatina anginosa they are often covered by a yellowish white exudation. In scarlatina maligna we have in addition ashy-coloured sloughs, and some of these sloughs go so deep into the tonsils that, when they break up, we have sometimes an artery of considerable size opened, causing alarming and dangerous hæmorrhage. In these forms pain of the throat is so great that deglutition is performed with great difficulty, and in some cases it cannot be performed at all. Secretion is greatly increased, so that it flows out of both mouth and nose, its character being so much altered that it irritates and excoriates the parts over which it flows.

I believe that in some of the zymotic diseases, where the poison can be reabsorbed, the disease is aggravated and prolonged. In enteric fever I have observed that where there was diarrhœa-although otherwise this indicates greater severity in the general and local symptomsthe course of the fever was generally shorter than where there was very little or none. The diarrhoea hurried the poison out of the bowel; but where there was no purging, or very little, it remained to be absorbed, prolonging the fever.

After these general remarks, I will now call your attention to some special points in the pathology of scarlet fever and the treatment.

A poison enters the blood, capable of extensive multiplication. After a shorter or longer period the tonsils and parts already mentioned, by some physiological law, take on the process of eliminating the poison, producing the local symptoms already stated. A great part of the eliminated poison must be swallowed, to be reabsorbed by the stomach, intensifying and prolonging the disease. In connection with this view, I will state a pathological condition of the stomach, which may, in part, be caused by the poison passing from the throat and other parts into it. 